\title{
Analytical Upper Bound for the Error on the Discretization of Uncertain Linear Systems by using the Tensor Product Model Transformation
}

\author{
Víctor C. da S. Campos ${ }^{1}$, Márcio F. Braga ${ }^{2}$, Luciano Frezzatto ${ }^{1}$ \\ Electronic Engineering Department, Engineering School, Federal University of \\ Minas Gerais (Universidade Federal de Minas Gerais - UFMG), Avenida Antônio \\ Carlos, 6627, CEP 31270-901, Belo Horizonte-MG, Brazil \\ \{kozttah,lfrezzatto\}@ufmg.br
}

Electrical Engineering Department, Exact and Applied Sciences Institute (ICEA), Federal University of Ouro Preto (Universidade Federal de Ouro Preto - UFOP), Rua Trinta e Seis, 115, CEP 35931-008, João Monlevade-MG, Brazil mfbraga@ufop.edu.br

\footnotetext{
Abstract: This work provides analytical upper bounds on the discretization error of uncertain linear systems. The Tensor Product Model Transformation is used to approximate the derived discretized system, with a reduced number of vertices. Digital state feedback controllers are then designed for the discretized system, for comparison to other available work in the current literature.
}

Keywords: Tensor Product Model Transformation; Discretization; Uncertain Linear Systems

\section{Introduction}

The technological development of high-performance computers and microprocessors allows for the usage of digital controllers [1], [2]. In real world applications, digital controllers have been widely used to control dynamical continuous-time systems, mainly due to their multiply advantages, such as, lower power consumption and fabrication costs, improved flexibility, ease in reprogramming the same device to deal with different control strategies and also implementation of complex digital control laws, the possibility of developing of interfaces with users (including web interfaces) and last, but not least, greater reliability [1], [3]. 
There are three main techniques used to synthesize digital controllers [4], [5], [6]: (i) the emulation design approach, where a continuous-time controller is designed regardless of the sampling time and, then, the controller is sampled [7], [8]; (ii) the discrete-time approach, where the design is done from a discrete-time description of the process which represents its behavior only in the sampling times, which means that the intersampling behavior are neglected [9], [10]; and, finally, (iii) the sampled-data design, where the acquired controller is synthesized based on a discrete-time model that takes into account the system behavior at the sampling and also in the intersampling times [11], [12].

The discretized version of the continuous-time system can be obtained by employing, for instance, the Taylor series expansion to deal with the exponential of the system dynamic matrix. Nevertheless, such technique can be directly applied only for systems without uncertainties. However, it is well known that real systems are usually affected by uncertainties which denote, for instance, neglected dynamics, external disturbances, unknown parameters, noise associated with the collected information or measurements, or the inaccuracy of sensors and actuators [13], making the discretization procedure of an uncertain system, which requires to compute the exponential of an uncertain matrix, a hard problem to deal with. To overcome such challenge, several numerical strategies have been used, such as Chebyshev quadrature formula and internal arithmetic, Jordan decomposition, or the Cayley-Hamilton theorem [14], [15], or, as more frequently found, a firstorder Taylor series expansion technique [16], [17], [18]. All of these methodologies can be employed only for systems with a small number of vertices and, especially, the latter, yields an inaccurate discrete-time model, mainly for larger sampling times.

A more recent result [9], uses a technique based on a Taylor series expansion of a fixed order to obtain the discrete-time representation of the continuous-time system, whose discrete-time model is composed of homogeneous polynomial matrices plus an additive norm-bounded term that represents the discretization residual error. Albeit, such procedure produces a more precise description of the systems dynamics, as a drawback, the discrete-time representation depends on multiple indexes increasing the number of Linear Matrix Inequalities (LMIs) to be solved, in the synthesis conditions, as the chosen order augments. In order to avoid the aforementioned problem, the work in [19] proposed an approach based on a grid of the possible values for the matrix exponential function and an application of the tensor product model transformation technique to acquire a suitable polytopic model, reducing the number of LMIs to be solved. However, the error committed by the discretization technique is ignored.

The Tensor Product Model Transformation (TPMT) [20], [21], [22], [23], [24], [25] is a numerical technique that allows one to extract a convex representation, similar to a Takagi-Sugeno (TS) representation for a quasi-Linear Parameter Varying representation of a dynamical system. It makes use of the Higher-Order Singular Value Decomposition (HOSVD) to numerically extract a meaningful 
representation from a sampled representation of a function over a grid of possible values. This convex representation allows the use of readily available Linear Matrix Inequality (LMI) conditions to be used for the synthesis of nonlinear systems in a systematic approach [24].

Several works in the literature focused on different applications of the TPMT to different control problems, and of special note is the work in [19], in which the authors made use of the Tensor Product Model Transformation to find a convex representation for the discretization of Uncertain Linear Systems. However, their work does not take into account the error made on this discretization procedure by using a grid of possible values for the discrete-time representation of the uncertain system. In that regard, this work extends the ideas introduced in [19], providing an analytical upper bound for the residual error norm and a discrete-time polytopic model for the continuous-time uncertain system.

\section{Notation}

In this paper, lowercase variables represent scalars, lowercase boldface variables represent column vectors, uppercase variables represent matrices and calligraphic uppercase variables represent tensors. $S^{T}$ denotes the transpose of matrix $S, Q>0$ $(Q \geq 0)$ indicates that matrix $Q$ is positive definite (positive semi-definite), and $\star$ indicates terms that can be inferred from symmetry on a symmetric matrix. $\mathcal{L} \times_{n} U$ represents the $\mathrm{n}$-mode product between tensor $\mathcal{L}$ and matrix $U$. In order to get acquainted with the multilinear algebra operations used in this paper, we refer the reader to [20], [27].

\section{Discretization Strategy}

Consider the uncertain linear system described by the polytopic model

$\dot{\boldsymbol{x}}=A(\boldsymbol{\alpha}) \boldsymbol{x}+B(\boldsymbol{\alpha}) \boldsymbol{u}=\sum_{i=1}^{r} \alpha_{i}\left(A_{i} \boldsymbol{x}+B_{i} \boldsymbol{u}\right)$

with $\boldsymbol{x} \in \mathbb{R}^{n}$ the system's states, $\boldsymbol{u} \in \mathbb{R}^{m}$ the control inputs, $A_{i} \in \mathbb{R}^{n \times n}$ and $B_{i} \in$ $\mathbb{R}^{n \times m}$ the system matrices and $\alpha_{i}$ the uncertain convex weights of the model, such that:

$\alpha_{i} \in[0,1], \sum_{i=1}^{r} \alpha_{i}=1$.

In this paper, our aim is to find an approximate uncertain discrete time polytopic model, for this system, described by:

$$
\begin{aligned}
\boldsymbol{x}_{\boldsymbol{k}+\mathbf{1}} & =(\hat{A}(\boldsymbol{\beta})+\Delta A) \boldsymbol{x}_{\boldsymbol{k}}+(\hat{B}(\boldsymbol{\beta})+\Delta B) \boldsymbol{u}_{\boldsymbol{k}} \\
& =\sum_{i=1}^{\hat{r}} \beta_{i}\left(\left(\hat{A}_{i}+\Delta A\right) \boldsymbol{x}_{\boldsymbol{k}}+\left(\hat{B}_{i}+\Delta B\right) \boldsymbol{u}_{\boldsymbol{k}}\right)
\end{aligned}
$$

with $\hat{r}$ the number of linear models composing the uncertain discretized system, $\beta_{i}$ the unknown convex weights with: 
$\beta_{i} \in[0,1], \sum_{i=1}^{\hat{r}} \beta_{i}=1$

and $\Delta A$ and $\Delta B$ norm bounded uncertainties with:

$\|\Delta A\|_{2} \leq \eta_{a}, \quad\|\Delta B\|_{2} \leq \eta_{b}$.

By considering that the uncertain convex weights $\alpha_{i}$ are constant over time and that the control inputs are constant over the sampling period, the system described in (1) can be exactly discretized by:

$\boldsymbol{x}_{\boldsymbol{k}+\mathbf{1}}=e^{A(\boldsymbol{\alpha}) \tau} \boldsymbol{x}_{\boldsymbol{k}}+\int_{0}^{\tau}\left(e^{A(\boldsymbol{\alpha}) t} d t\right) B(\boldsymbol{\alpha}) \boldsymbol{u}_{\boldsymbol{k}}$

with $\tau$ the sampling period. Similarly to [19], our problem can be restated as finding a convex representation for:

$\hat{A}(\boldsymbol{\alpha})=e^{A(\boldsymbol{\alpha}) \tau}, \hat{B}(\boldsymbol{\alpha})=\int_{0}^{\tau}\left(e^{A(\boldsymbol{\alpha}) t} d t\right) B(\boldsymbol{\alpha})$,

and the Tensor Product Model Transformation (TPMT) [20], [21], [22], [23] can be employed to this end. Unlike [19] though, we explicitly consider the discretization error introduced by the sampling step of the TPMT, which are represented by $\Delta A$ and $\Delta B$ in (3). The main contribution in this paper can then be understood as analytical upper bounds on $\eta_{a}$ and $\eta_{b}$.

\subsection{Tensor Product Model Transformation (TPMT)}

In order to find a representation with the smallest $\hat{r}$ (or the smallest number of linear systems composing the uncertain model), we define the matrix function:

$H(\boldsymbol{\alpha})=\left[\begin{array}{ll}\hat{A}(\boldsymbol{\alpha}) & \hat{B}(\boldsymbol{\alpha})\end{array}\right]$

which will be approximated by the Tensor Product Model Transformation. This approach is common in the TPMT literature and allows for a smaller number of vertices found in the end, when compared against approximating the matrix functions separately and joining the convex models found afterwards.

The TPMT can usually be divided into four steps: sampling, Higher Order Singular Value Decomposition (HOSVD), Convex Hull Manipulation and Interpolation [26].

\subsubsection{Sampling}

If done on the usual approach, the sampling step would be performed by defining a regular sampling grid over the hyperrectangular domain $\alpha \in[0,1]^{r}$ and, if we considered sampling each $\alpha_{i}$ with $p$ samples, storing it on a tensor $\mathcal{H} \in$ $\mathbb{R}^{p \times \ldots \times p \times n \times(n+m)}$.

This approach is usually employed with Linear Parameter Varying (LPV) and Takagi-Sugeno (TS) models, since it allows for a special structure in which the resulting weights can be decomposed as functions of a single scalar variable. 
When such structure on the weighting functions is not needed, an approach similar to the 1-level Nested Tensor Product Model Transformation (NTPMT) [21] could be used instead, resulting in a tighter model with a smaller number of linear systems composing the desired model.

We make use, instead, of the approach proposed in [19] since we have a particular structure on our domain. From (2) we know that:

$\alpha_{r}=1-\sum_{i=1}^{r-1} \alpha_{i}$

and we need only to sample the first $r-1$ dimensions of $\boldsymbol{\alpha}$. In addition to this, we only consider valid samples as being those for which:

$\sum_{i=1}^{r-1} \alpha_{i} \leq 1$.

Inspired by the 1-level NPTMT, we store the valid samples on a tensor $\mathcal{H} \in$ $\mathbb{R}^{\kappa \times n \times(n+m)}$, with $\kappa$ the number of valid samples taken and $\mathcal{H}_{i j k}$ the element in row $j$ and column $k$ from sample $i$.

With this sampling, the matrix function can be represented as:

$H(\boldsymbol{\alpha})=\mathcal{H} \times_{1} \boldsymbol{w}^{T}(\boldsymbol{\alpha})+\Delta H$

with $\boldsymbol{w}^{\boldsymbol{T}}(\boldsymbol{\alpha})$ an interpolation function that assigns a weight to each sample depending on the value of $\boldsymbol{\alpha}$ and $\Delta H$ the interpolation/grid sampling error. While any interpolation strategy that yields convex weights could be used, like a finite element interpolation for instance, in this work we consider a nearest neighbor, or piecewise constant, interpolation to derive an upper bound on the norm of the part that compose $\Delta H(\Delta A$ e $\Delta B)$.

\subsubsection{Higher Order Singular Value Decomposition (HOSVD)}

By making use of the HOSVD [27] along the first direction of $\mathcal{H}$, it can be rewritten as:

$\mathcal{H}=\mathcal{L} \times{ }_{1} U_{1}$

with $\mathcal{L} \in \mathbb{R}^{q \times n \times(n+m)}, U_{1} \in \mathbb{R}^{\kappa \times q}$, and the equality is ensured in the equation above only on the cases in which no nonzero higher order singular values are discarded. In case nonzero higher order singular values are discarded, the approach presented in [26] can be used to find an extra uncertainty that needs to be considered in $\Delta H$.

\subsubsection{Convex Hull Manipulation}

In order for us to retrieve an interesting convex representation for function $H(\alpha)$, it is necessary to impose some special properties upon matrix $U_{1}$. The following properties are common in the TPMT literature:

- Sum Normalization (SN): for every row of $U_{1}$, the sum of its columns is equal to one 
- Non Negative (NN): every element of $U_{1}$ is nonnegative

- Inverse Normalized (INO): the minimum of every column of $U_{1}$ is the same and is equal to zero

- Relaxed Normalized (RNO): the maximum of every column of $U_{1}$ is the same

- Close to Normalized (CNO): the simplex formed by the unitary vectors is the smallest volume simplex that covers the vectors formed by the rows of $U_{1}$

The SN and NN properties are the bare minimum to ensure that we retrieve a convex representation, but usually do not generate interesting models by themselves. The other properties are usually employed to guarantee that a "tight" representation is found, meaning that, in this case, they aim at representing the uncertain system with a small set.

In the examples presented later in this work, we make use of the SN-NN transformation [22], followed by the RNO-INO transformation [28] and the CNO transformation.

\subsubsection{Interpolation}

If the combined transformations are such that:

$\widehat{U}_{1}=U_{1} T_{1} \Rightarrow U_{1}=\widehat{U}_{1} T_{1}^{-1}$

with $\widehat{U}_{1}$ the matrix with the desired properties and $T_{1}$ the nonsingular matrix that transforms the original matrix. Then by combining (11), (12) and (13) we get that

$$
\begin{aligned}
& H(\boldsymbol{\alpha})=\left(\mathcal{L} \times_{1} U_{1}\right) \times_{1} \boldsymbol{w}^{T}(\boldsymbol{\alpha})+\Delta H \\
& =\left(\mathcal{L} \times_{1} \widehat{U}_{1} T_{1}^{-1}\right) \times_{1} \boldsymbol{w}^{T}(\boldsymbol{\alpha})+\Delta H \\
& =\left(\mathcal{L} \times{ }_{1} T_{1}^{-1}\right) \times_{1} \boldsymbol{w}^{T}(\boldsymbol{\alpha}) \widehat{U}_{1}+\Delta H \\
& =\hat{\mathcal{L}} \times{ }_{1} \widehat{\boldsymbol{w}}^{T}(\boldsymbol{\alpha})+\Delta H \\
& =\sum_{i=1}^{\hat{r}} \beta_{i}\left[\begin{array}{ll}
\hat{A}_{i} & \hat{B}_{i}
\end{array}\right]+\Delta H
\end{aligned}
$$

with $\beta_{i}=w_{i}(\boldsymbol{\alpha}), \hat{A}_{i_{j k}}=\hat{\mathcal{L}}_{i j k}$ and $\hat{B}_{i_{j k}}=\hat{\mathcal{L}}_{i j(k+n)}$. Note that, even though we present a form to calculate $\beta_{\mathrm{I}}$ since the values of $\boldsymbol{\alpha}$ are unknown, and we consider the values of $\beta_{\mathrm{I}}$ to be unkown as well, they need not be determined.

\subsection{Analytical Upper Bound on the Grid Sampling Step}

By comparing (14) and (3), we get that the error in (14) can be rewritten as:

$\Delta H=\left[\begin{array}{ll}\Delta A & \Delta B\end{array}\right]$ 
Note that, since we are using a nearest neighbour interpolation these errors can be taken as the error in this kind of interpolation on the sampling grid. As such, consider that $\boldsymbol{\alpha}$ represents any point on the domain, while $\boldsymbol{\alpha}_{\boldsymbol{g}}$ represents the nearest point on the grid. We get that the interpolation errors can be written as:

$\Delta A=e^{A(\boldsymbol{\alpha}) \tau}-e^{A\left(\boldsymbol{\alpha}_{\boldsymbol{g}}\right) \tau}$

$\Delta B=\int_{0}^{\tau}\left(e^{A(\boldsymbol{\alpha}) t} d t\right) B(\boldsymbol{\alpha})-\int_{0}^{\tau}\left(e^{A\left(\boldsymbol{\alpha}_{\boldsymbol{g}}\right) t} d t\right) B\left(\boldsymbol{\alpha}_{\boldsymbol{g}}\right)$

Since $\boldsymbol{\alpha}_{\boldsymbol{g}}$ represents the nearest point on the grid, (16) can be rewritten as:

$\Delta A=e^{A\left(\boldsymbol{\alpha}_{\boldsymbol{g}}\right) \tau+A\left(\boldsymbol{\delta}_{\boldsymbol{\alpha}}\right) \tau}-e^{A\left(\boldsymbol{\alpha}_{\boldsymbol{g}}\right) \tau}$

with

$A\left(\boldsymbol{\delta}_{\alpha}\right)=\sum_{i=1}^{r}\left(\alpha_{i}-\alpha_{g_{i}}\right) A_{i}$

From the definition of the matrix exponential with its Taylor series, we get that:

$$
\begin{aligned}
\Delta A & =\sum_{i=0}^{\infty}\left(\frac{\left(A\left(\boldsymbol{\alpha}_{\boldsymbol{g}}\right) \tau+A\left(\boldsymbol{\delta}_{\boldsymbol{\alpha}}\right) \tau\right)^{i}}{i !}-\frac{\left(A\left(\boldsymbol{\alpha}_{\boldsymbol{g}}\right) \tau\right)^{i}}{i !}\right) \\
& =\sum_{i=0}^{\infty} \frac{1}{i !}\left(\sum_{k=0}^{i}\left(\begin{array}{c}
i \\
k
\end{array}\right)\left(A\left(\boldsymbol{\alpha}_{\boldsymbol{g}}\right) \tau\right)^{k}\left(A\left(\boldsymbol{\delta}_{\boldsymbol{\alpha}}\right) \tau\right)^{i-k}-\left(A\left(\boldsymbol{\alpha}_{\boldsymbol{g}}\right) \tau\right)^{i}\right) \\
& =\sum_{i=0}^{\infty} \frac{1}{i !}\left(\sum_{k=0}^{i-1}\left(\begin{array}{c}
i \\
k
\end{array}\right)\left(A\left(\boldsymbol{\alpha}_{\boldsymbol{g}}\right) \tau\right)^{k}\left(A\left(\boldsymbol{\delta}_{\boldsymbol{\alpha}}\right) \tau\right)^{i-k}\right)
\end{aligned}
$$

By taking the norm on both sides, we can write that:

$$
\|\Delta A\|_{2} \leq \eta_{a}=\sum_{i=0}^{\infty} \sum_{k=0}^{i-1} \frac{1}{i !}\left(\begin{array}{c}
i \\
k
\end{array}\right)\left\|A\left(\alpha_{g}\right)\right\|_{2}^{k}\left\|A\left(\delta_{\alpha}\right)\right\|_{2}^{i-k} \tau^{i}
$$

Once again, since $\boldsymbol{\alpha}_{\boldsymbol{g}}$ represents the nearest point on the grid, (17) can be rewritten as:

$\Delta B=\int_{0}^{\tau}\left(e^{A(\boldsymbol{\alpha}) t}-e^{A\left(\boldsymbol{\alpha}_{\boldsymbol{g}}\right) t} d t\right) B(\boldsymbol{\alpha})+\int_{0}^{\tau}\left(e^{A\left(\boldsymbol{\alpha}_{\boldsymbol{g}}\right) t} d t\right)\left(B\left(\boldsymbol{\alpha}_{\boldsymbol{g}}\right)-B(\boldsymbol{\alpha})\right)$

By taking the norm on both sides of (22) and making use of the triangular inequality, we get that:

$$
\begin{aligned}
\|\Delta B\|_{2} & \leq\left\|\int_{0}^{\tau}\left(e^{A(\boldsymbol{\alpha}) t}-e^{A\left(\boldsymbol{\alpha}_{\boldsymbol{g}}\right) t} d t\right) B(\boldsymbol{\alpha})\right\|_{2} \\
& +\left\|\int_{0}^{\tau}\left(e^{A\left(\boldsymbol{\alpha}_{\boldsymbol{g}}\right) t} d t\right)\left(B\left(\boldsymbol{\delta}_{\boldsymbol{\alpha}}\right)\right)\right\|_{2}
\end{aligned}
$$

with

$B\left(\boldsymbol{\delta}_{\alpha}\right)=\sum_{i=1}^{r}\left(\alpha_{i}-\alpha_{g_{i}}\right) B_{i}$

The first term on the right hand side of (23) can be upper bounded, by making use of the developments in (20), by: 


$$
\begin{aligned}
& \left\|\int_{0}^{\tau}\left(e^{A(\boldsymbol{\alpha}) t}-e^{A\left(\boldsymbol{\alpha}_{\boldsymbol{g}}\right) t} d t\right) B(\boldsymbol{\alpha})\right\|_{2} \leq \\
& \int_{0}^{\tau} \sum_{i=0}^{\infty} \frac{t^{i}}{i !}\left(\sum_{k=0}^{i-1}\left(\begin{array}{c}
i \\
k
\end{array}\right)\left\|A\left(\boldsymbol{\alpha}_{\boldsymbol{g}}\right)\right\|_{2}^{k}\left\|A\left(\boldsymbol{\delta}_{\boldsymbol{\alpha}}\right)\right\|_{2}^{i-k}\right) d t\|B(\boldsymbol{\alpha})\|_{2} \leq \\
& \sum_{i=0}^{\infty} \frac{t^{i+1}}{(i+1) !}\left(\sum_{k=0}^{i-1}\left(\begin{array}{c}
i \\
k
\end{array}\right)\left\|A\left(\boldsymbol{\alpha}_{\boldsymbol{g}}\right)\right\|_{2}^{k}\left\|A\left(\boldsymbol{\delta}_{\boldsymbol{\alpha}}\right)\right\|_{2}^{i-k}\right)\|B(\boldsymbol{\alpha})\|_{2}
\end{aligned}
$$

whereas the second term can be upper bounded by:

$$
\begin{aligned}
& \left\|\int_{0}^{\tau}\left(e^{A\left(\boldsymbol{\alpha}_{\boldsymbol{g}}\right) t} d t\right)\left(B\left(\boldsymbol{\delta}_{\boldsymbol{\alpha}}\right)\right)\right\|_{2} \leq \int_{0}^{\tau}\left\|e^{A\left(\boldsymbol{\alpha}_{\boldsymbol{g}}\right) t}\right\|_{2}\left\|B\left(\boldsymbol{\delta}_{\boldsymbol{\alpha}}\right)\right\|_{2} d t \leq \\
& \int_{0}^{\tau} e^{\left\|A\left(\boldsymbol{\alpha}_{\boldsymbol{g}}\right)\right\|_{2} t}\left\|B\left(\boldsymbol{\delta}_{\boldsymbol{\alpha}}\right)\right\|_{2} d t=\frac{\left\|B\left(\boldsymbol{\delta}_{\boldsymbol{\alpha}}\right)\right\|_{2}}{\left\|A\left(\boldsymbol{\alpha}_{\boldsymbol{g}}\right)\right\|_{2}}\left(e^{\left\|A\left(\boldsymbol{\alpha}_{\boldsymbol{g}}\right)\right\|_{2} \tau}-1\right)
\end{aligned}
$$

By considering (25) and (26), we can write that:

$\|\Delta B\|_{2} \leq \eta_{b}$

with

$$
\begin{aligned}
& \eta_{b}=\sum_{i=0}^{\infty} \frac{t^{i+1}}{(i+1) !}\left(\sum_{k=0}^{i-1}\left(\begin{array}{c}
i \\
k
\end{array}\right)\left\|A\left(\boldsymbol{\alpha}_{\boldsymbol{g}}\right)\right\|_{2}^{k}\left\|A\left(\boldsymbol{\delta}_{\boldsymbol{\alpha}}\right)\right\|_{2}^{i-k}\right)\|B(\boldsymbol{\alpha})\|_{2}+ \\
& \int_{0}^{\tau} e^{\left\|A\left(\boldsymbol{\alpha}_{\boldsymbol{g}}\right)\right\|_{2} t}\left\|B\left(\boldsymbol{\delta}_{\boldsymbol{\alpha}}\right)\right\|_{2} d t=\frac{\left\|B\left(\boldsymbol{\delta}_{\boldsymbol{\alpha}}\right)\right\|_{2}}{\left\|A\left(\boldsymbol{\alpha}_{\boldsymbol{g}}\right)\right\|_{2}}\left(e^{\left\|A\left(\boldsymbol{\alpha}_{\boldsymbol{g}}\right)\right\|_{2} \tau}-1\right)
\end{aligned}
$$

Equations (21) and (28) allow us to find an upper bound on the grid sampling step and can be used to determine bounds for the norm-bounded uncertainty in (3). In order to make use of these equations, though, we need to be able to calculate $\left\|A\left(\boldsymbol{\alpha}_{\boldsymbol{g}}\right)\right\|_{2},\left\|B\left(\boldsymbol{\alpha}_{\boldsymbol{g}}\right)\right\|_{2},\left\|A\left(\boldsymbol{\delta}_{\boldsymbol{\alpha}}\right)\right\|_{2}$ and $\left\|B\left(\boldsymbol{\delta}_{\boldsymbol{\alpha}}\right)\right\|_{2}$.

To do so, we consider that the sampling grid is such that:

$\left|\alpha_{i_{\ell}}-\alpha_{g_{i_{\ell}}}\right| \leq h$

By using this limit, together with the fact that:

$\sum_{i=1}^{r}\left(\alpha_{i}-\alpha_{g_{i}}\right)=0$

and that the intersection of a polytope and a hyperplane is always a polytope, we can write:

$\boldsymbol{\alpha}-\boldsymbol{\alpha}_{\boldsymbol{g}}=\sum_{k=1}^{n_{v}} \gamma_{k} \boldsymbol{v}_{\boldsymbol{k}}$

with $\boldsymbol{v}_{\boldsymbol{k}}$ the vertices of the intersection from the $[-h, h]^{r}$ polytope with the zerosum hyperplane and

$\gamma_{k} \in[0,1], \sum_{k=1}^{n_{v}} \gamma_{k}=1$

Finally, with these definitions, we have that:

$\left\|A\left(\boldsymbol{\alpha}_{\boldsymbol{g}}\right)\right\|_{2} \leq \max _{i}\left\|A_{i}\right\|_{2}$
$\left\|B\left(\boldsymbol{\alpha}_{\boldsymbol{g}}\right)\right\|_{2} \leq \max _{i}\left\|B_{i}\right\|_{2}$ 


$$
\begin{aligned}
& \left\|A\left(\boldsymbol{\delta}_{\boldsymbol{\alpha}}\right)\right\|_{2} \leq \max _{k}\left\|\sum_{i=1}^{r} v_{k i} A_{i}\right\|_{2} \\
& \left\|B\left(\boldsymbol{\delta}_{\boldsymbol{\alpha}}\right)\right\|_{2} \leq \max _{k}\left\|\sum_{i=1}^{r} v_{k i} B_{i}\right\|_{2}
\end{aligned}
$$

\section{Illustrative Examples}

To illustrate the advantages of the proposed discretization procedure, we synthesize digital state feedback controllers based on the derived discretized model. For that, we adapted the controller design condition provided in [30], which is given in the following theorem.

Theorem 1 (adapted from [30]). For given $\eta_{A}$ and $\eta_{B}$, if there exist positive definite matrices $P_{i}=P_{i}^{T} \in \mathbb{R}^{n \times n}, i=1, \ldots, \hat{r}$, matrices $G \in \mathbb{R}^{n \times n}, X \in \mathbb{R}^{m \times n}$, and a scalar $\mu$ such that:

$\left[\begin{array}{cccc}P_{i}-G^{T}-G & \star & \star & \star \\ \hat{A}_{i} G+\hat{B}_{i} X & -P_{i}+\mu\left(\eta_{A}^{2}+\eta_{B}^{2}\right) I & \star & \star \\ G & 0 & -\mu I & \star \\ X & 0 & 0 & -\mu I\end{array}\right]<0, \forall i$

then there exists a digital state feedback controller given by $K=X G^{-1}$ that asymptotically stabilizes system (1).

We compare our discretization procedure with the ones of [10] and [30] in terms of the maximum sampling period and the bounds of the discretization error. Notice that our approach and the one of [30] provide distinct upper bounds for matrices $\hat{A}(\beta)$ and $\hat{B}(\beta)$, whereas the approach of [10] provides a single upper bound for both matrices. Two numerical examples are provided to this end. It is important to emphasize that all comparisons are performed among methods that provide a theoretical bound for the system's discretization error.

Example 1. Consider a linearized inverted pendulum on cart model given by equation (1) with:

$A=\left[\begin{array}{cccc}0 & 1 & 0 & 0 \\ 0 & -\frac{b\left(\alpha_{1}\right)}{M\left(\alpha_{2}\right)} & -\frac{m g}{M\left(\alpha_{2}\right)} & 0 \\ 0 & 0 & 0 & 1 \\ 0 & \frac{b\left(\alpha_{1}\right)}{M\left(\alpha_{2}\right) l} & \frac{\left(M\left(\alpha_{2}\right)+m\right) g}{M\left(\alpha_{2}\right) l} & 0\end{array}\right], \quad B=\left[\begin{array}{c}0 \\ \frac{1}{M\left(\alpha_{2}\right)} \\ 0 \\ -\frac{1}{M\left(\alpha_{2}\right) l}\end{array}\right]$

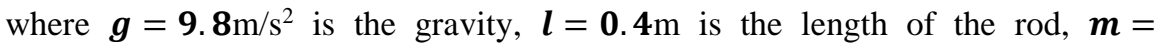
$0.11 \mathrm{~kg}$ is the mass of the pendulum (assumed concentrated at the end of the rod), $\boldsymbol{b}\left(\boldsymbol{\alpha}_{1}\right) \in[0.04750 .0525] \mathrm{Ns} / \mathrm{m}$ is the friction coefficient of the cart, and $\boldsymbol{M}\left(\boldsymbol{\alpha}_{2}\right) \in[\mathbf{0 . 8 9 6} 1.344] \mathrm{kg}$ is the mass of the cart.

Our aim in this example is determine the maximum discretization period that ensures the closed loop system (with a digital controller) is asymptotically stable. 
Applying the conditions of Theorem 1 with 111 points and a tolerance of $\mathbf{1 0}^{-\mathbf{6}}$ provided a maximum period of $\boldsymbol{\tau}=\mathbf{1 7 8} \mathrm{ms}$ with error bounds for the discretization procedure of $\boldsymbol{\eta}_{\boldsymbol{A}}=\mathbf{0 . 0 0 4 3}$ and $\boldsymbol{\eta}_{\boldsymbol{B}}=\mathbf{0 . 0 0 1 2}$. Contrast this sampling time with the maximum ones obtained by the methods of [30], $\boldsymbol{\tau}=$ 103ms, and of [10], $\boldsymbol{\tau}=\mathbf{1 7 8} \mathrm{ms}$, with error bounds of $\boldsymbol{\eta}_{\boldsymbol{A}}=\mathbf{0 . 0 4 5 9}$ and $\boldsymbol{\eta}_{\boldsymbol{B}}=$ $\mathbf{0 . 0 0 5 1}$, and $\boldsymbol{\eta}_{\boldsymbol{A}}=\boldsymbol{\eta}_{\boldsymbol{B}}=\mathbf{0 . 0 4 7 3}$, respectively. For the method of [30], a truncated $\mathbf{8}^{\text {th }}$ order Taylor series expansion was adopted, polynomial matrices of degree 8 and $\boldsymbol{L}=\mathbf{2 0 0}$ (a parameter used by [30] to calculate the discretization error); whereas, for the approach of [10], a truncated $\mathbf{1 0}^{\text {th }}$ order Taylor series expansion is considered with affine-dependent optimization variables. Notice that our discretization period is about 1.7 times greater than the one of [30] whereas it is the same as [10]. Nevertheless, our approach does not require determining the Taylor series expansion of each matrix in order to obtain a discretized model and, furthermore, the analytic upper bound for the discretization error is about 10 times smaller than in the other methods. Table 1 summarizes the results attained for each aforementioned method.

Table 1

Summary of the discretization procedures results for the system of Example 1

\begin{tabular}{|c|c|c|c|}
\hline Method & Max. Samp. Time & $\boldsymbol{\eta}_{\boldsymbol{A}}$ & $\boldsymbol{\eta}_{\boldsymbol{B}}$ \\
\hline Theorem 1 & $178 \mathrm{~ms}$ & 0.0043 & 0.0012 \\
\hline$[30]$ & $103 \mathrm{~ms}$ & 0.0463 & 0.0051 \\
\hline$[10]$ & $178 \mathrm{~ms}$ & \multicolumn{2}{|c|}{0.0473} \\
\hline
\end{tabular}

To further illustrate the performance of our approach, a time simulation of the continuous system with the digital controller is performed and the attained results are depicted in Figure 1 and Figure 2. Starting from an initial condition of $\boldsymbol{x}_{\mathbf{0}}=$ $\left[\begin{array}{llll}\mathbf{0} & \mathbf{0} & \boldsymbol{\pi} / \mathbf{6} & \mathbf{0}\end{array}\right]^{T}$, the simulation was performed for 20 seconds and the controller synthesized for the largest attained sampling time was adopted. Notice that the closed-loop inverted pendulum system stabilizes in about 10 seconds and that the amplitude of the control signal presents reasonable bounds for a real implementation.

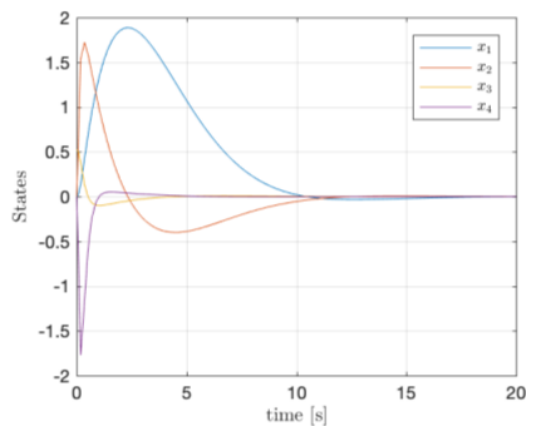

Figure 1

Evolution of the inverted pendulum states 


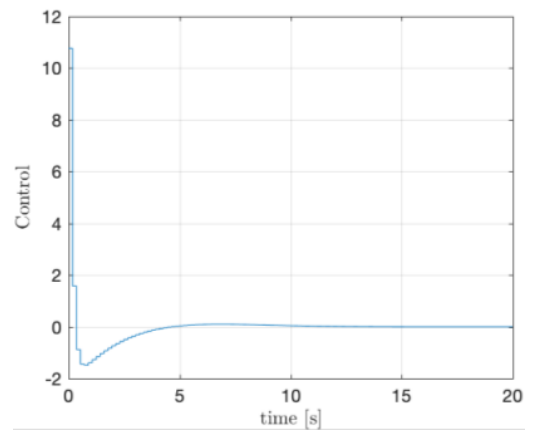

Figure 2

Digital control signal applied to the inverted pendulum system

Example 2. In this example an open-loop stable system is considered, which is a simplified two-mass-spring system as proposed in [31] described by equation (1) with the following matrices:

$A=\left[\begin{array}{cccc}0 & 0 & 1 & 0 \\ 0 & 0 & 0 & 1 \\ -\frac{k(\alpha)}{m_{1}} & \frac{k(\alpha)}{m_{1}} & 0 & 0 \\ \frac{k(\alpha)}{m_{2}} & -\frac{k(\alpha)}{m_{2}} & 0 & 0\end{array}\right], \quad B=\left[\begin{array}{c}0 \\ 0 \\ 1 \\ \frac{m_{1}}{0}\end{array}\right]$

where $\boldsymbol{m}_{\mathbf{1}}=\boldsymbol{m}_{\mathbf{2}}=\mathbf{1} \mathrm{kg}$ are the masses of carts 1 and 2, respectively, and $\boldsymbol{k}(\boldsymbol{\alpha}) \in$ $\left[\begin{array}{ll}0.5 & 2.0\end{array}\right] \mathrm{N} / \mathrm{m}$ is the spring constant.

The methods of [10] and [30] (using a truncated $\mathbf{1 0}^{\text {th }}$ order Taylor series expansion) are once again applied to the above system and the attained results are compared to our proposed approach. These results are reported in Table 2. From the presented data, one can notice that the maximum sampling time attained by our approach is higher than both [10] and [30] and besides the discretization error is about 10 times smaller than the one provided by [10] and 4 times smaller than the one of [30].

Table 2

Maximum sampling times and analytical error bounds for Theorem 1 and the methods of [10] and [30]

\begin{tabular}{|c|c|c|c|}
\hline Method & Max. Samp. Time & $\boldsymbol{\eta}_{\boldsymbol{A}}$ & $\boldsymbol{\eta}_{\boldsymbol{B}}$ \\
\hline Theorem 1 & 1.217 & 0.0266 & 0.0056 \\
\hline$[30]$ & 1.018 & 0.1909 & 0.0477 \\
\hline$[10]$ & 1.112 & \multicolumn{2}{|c|}{0.0987} \\
\hline
\end{tabular}

\section{Conclusions and Future Works}

In this work, we derived an analytical upper bound on the discretization approach proposed in [19]. In order to do so, we utilized a nearest neighbor interpolation in the TPMT and performed manipulations around the definition of the matrix 
exponential function. The proposed bounds are considerably smaller than other analytical bounds available for other discretization approaches in the literature.

In the future, we aim to develop tighter bounds for this error, possibly by using different interpolation strategies. Another interesting development would be the use of the NTPMT [21] for the discretization and comparing the results against the learnings in this paper.

\section{Acknowledgement}

This study was financed in part by the Coordenação de Aperfeiçoamento de Pessoal de Nível Superior - Brasil (CAPES) - Finance Code 001, Conselho Nacional de Desenvolvimento Científico e Tecnológico (CNPq), Universidade Federal de Minas Gerais and Universidade Federal de Ouro Preto, process number 23109.003268/2017-47.

\section{References}

[1] T. Chen and B. A. Francis, "Optimal Sampled-Data Control Systems". London, UK: Springer-Verlag, 1995

[2] W. Zhang, M. S. Branicky, and S. M. Phillips. "Stability of networked control systems”. IEEE Control Systems Magazine, 21, 84-99, 2001

[3] S. Hara, Y. Yamamoto, and H. Fujioka, "Modern and classical analysis/ synthesis methods in sampled-data control - A brief overview with numerical examples,” Kobe, Japan, December 1996, pp. 1251-1256

[4] C. L. Phillips and H. T. Nagle. "Digital Control System Analysis and Design”, Vol. 3, Prentice-Hall, 1995

[5] S. Monaco and D. Normand-Cyrot, "Issues on nonlinear digital control". European Journal of Control, 7, 160-177, 2001

[6] D. Nešić and R. Postoyan, "Nonlinear sampled-data systems". In Encyclopedia of Systems and Control, 1-7, 2014

[7] G. F. Franklin, J. D. Powell, and M. L. Workman, "Digital control of dynamic systems". Addison-wesley Menlo Park, CA, 1998, Vol. 3

[8] L. S. Shieh, W. M. Wang, and J. S. H. Tsai, "Digital modelling and digital redesign of sampled-data uncertain systems," IEE Proceedings - Control Theory \& Applications, Vol. 142, No. 6, pp. 585-594, November 1995

[9] M. F. Braga, C. F. Morais, E. S. Tognetti, R. C. L. F. Oliveira, and P. L. D. Peres, "Discretisation and control of polytopic systems with uncertain sampling rates and network-induced delays," International Journal of Control, Vol. 87, No. 11, pp. 2398-2411, November 2014

[10] M. Jungers, G. S. Deaecto, and J. C. Geromel, "Bounds for the remainders of uncertain matrix exponential and sampled-data control of polytopic linear systems," Automatica, Vol. 82, pp. 202-208, 2017 
[11] L.-S. Hu, J. Lam, Y.-Y. Cao, and H.-H. Shao, "A linear matrix inequality (LMI) approach to robust $\mathrm{H} 2$ sampled-data control for linear uncertain systems," IEEE Transactions on Systemz, Man, and Cybernetics, Part B (Cybernetics), Vol. 33, No. 1, pp. 149-155, February 2003

[12] E. Gershon and U. Shaked, "Vertex-dependent approach to robust $\mathrm{H}_{\infty}$ control and estimation of stochastic discrete-time systems," IFACPapersOnLine, Vol. 48, No. 11, pp. 949-953, 2015

[13] J. Ackermann, "Robust control: Systems with uncertain physical parameters". London: Springer Verlag, 1993

[14] H. Su, J. Wang, and J. Chu, "Robust memoryless $\mathrm{H}_{\infty}$ control for uncertain linear time-delay systems," in Proceedings of the 1998 American Control Conference, Philadelphia, PA, USA, June 1998, pp. 3730-3731

[15] W. P. Heemels, N. van de Wouw, R. H. Gielen, M. C. F. Donkers, L. Hetel, S. Olaru, M. Lazar, J. Daafouz, and S. Niculescu, "Comparison of overapproximation methods for stability analysis of networked control systems," in Proceedings of the $13^{\text {th }}$ ACM International Conference on Hybrid Systems: Computation and Control, ser. HSCC'10. New York, NY, USA: ACM, 2010, pp. 181-190

[16] M. V. Kothare, V. Balakrishnan, and M. Morari, "Robust constrained model predictive control using linear matrix inequalities," Automatica, Vol. 32, No. 10, pp. 1361-1379, October 1996

[17] S. Lee and S. Won, "Model Predictive Control for linear parameter varying systems using a new parameter dependent terminal weighting matrix," IEICE Transactions on Fundamentals of Electronics, Communications and Computer Sciences, Vol. E89-A, No. 8, pp. 2166-2172, 2006

[18] N. Wada, K. Saito, and M. Saeki, "Model predictive control for linear parameter varying systems using parameter dependent Lyapunov function," IEEE Transactions on Circuits \& Systems II: Express Briefs, Vol. 53, No. 12, pp. 1446-1450, December 2006

[19] V. C. S. Campos, L. M. S. Vienna, M. F. Braga, "A tensor product model transformation approach to the discretization of uncertain linear systems". Acta Polytechnica Hungarica, Vol. 15, No. 3, pp. 31-53, 2018

[20] P. Baranyi, Y. Yam, and P. Várlaki, "Tensor Product Model Transformation in Polytopic Model-Based Control". Boca Raton: CRC Press, 2014

[21] Y. Yu, Z. Li, X. Liu, K. Hirota, X. Chen, T. Fernando, and H. H. Iu, “A nested tensor product model transformation". IEEE Transactions on Fuzzy Systems, 27(1), pp. 1-15, 2019 
[22] Y. Yam, P. Baranyi, and C.-T. Yang, "Reduction of fuzzy rule base via singular value decomposition," IEEE Transactions on Fuzzy Systems, Vol. 7, No. 2, pp. 120-132, 1999

[23] P. Baranyi, D. Tikk, Y. Yam, and R. J. Patton, "From differential equations to PDC controller design via numerical transformation", Computers in Industry, Vol. 51, No. 3, pp. 281-297, 2003

[24] P. Baranyi, "TP model transformation as a way to LMI-based controller design”, IEEE Transactions on Industrial Electronics, Vol. 51, No. 2, pp. 387-400, 2004

[25] P. Baranyi, "Extracting LPV and qLPV structures from state-space functions: a TP model transformation based framework". IEEE Transactions on Fuzzy Systems, Early Access (to appear), 2020

[26] V. C. da S. Campos, L. A. B. Tôrres, and R. M. Palhares, "Revisiting the TP model transformation: Interpolation and rule reduction". Asian Journal of Control, v. 17, n. 2, pp. 392-401, 2015

[27] L. De Lathauwer, B. De Moor, and J. Vandewalle, “A Multilinear Singular Value Decomposition," SIAM Journal on Matrix Analysis and Applications, Vol. 21, No. 4, p. 1253, 2000

[28] P. Varkonyi, D. Tikk, P. Korondi, and P. Baranyi, "A new algorithm for RNO-INO type tensor product model representation," in 2005 IEEE International Conference on Intelligent Engineering Systems, INES'05, IEEE, 2005, pp. 263-266

[29] V. C. S. Campos, F. Souza, L. A. B. Tôrres, and R. M. Palhares, "New stability conditions based on piecewise fuzzy Lyapunov functions and tensor product transformations," IEEE Transactions on Fuzzy Systems, Vol. 21, No. 4, pp. 748-760, 2013

[30] D. H. Lee and Y. H. Joo, "LMI-based Robust Sampled-data Stabilizationof Polytopic LTI Systems: A Truncated Power Series Expansion Approach”, International Journal of Control, Automation, and Systems, Vol. 13, No. 3, pp. 1-8, 2015

[31] B. Wie and D. S. Bernstein, "Benchmark Problems for Robust Control Design”, Journal of Guidance, Control, and Dynamics, Vol. 15, No. 5, pp. 1057-1059, 1992 